\title{
Significance of Lactate Levels in Vaginal Fluid with Time of Spontaneous Onset of Labour in Suspected Cases of Pre-Labour Rupture Of Membranes
}

\author{
Prof (Dr.) RizwanaHabib Kant ${ }^{1}$, Dr. Sabia Rashid ${ }^{2}$, Dr. Mahapara ${ }^{3}$, Dr. Saima \\ Salam $^{4}$, Dr. Preeti Sharma ${ }^{5}$ \\ ${ }^{1}$ Professor and $\mathrm{HOU}$ \\ 2, 3, 4, ${ }^{\text {Registrar }}$
}

\begin{abstract}
Background: Prelabour rupture of membranes is reported to occur in 2-25\% of all pregnancies. The condition is associated with fetal and maternal infections, cord complications and might be marker of imminent delivery. It is therefore of great importance to accurately confirm the diagnosis and determine the latency period between rupture of membranes and delivery for referral of patients to higher institutions with NICU facilities and thus improve the perinatal outcome. The lactate concentration in amniotic fluid is 4-6 times higher as compared with maternal and foetal blood and hence its level in vaginal fluid can be used to establish prelabour rupture of membranes and time of spontaneous onset of labour in these patients.
\end{abstract}

Objectives:

1. To diagnose prelabour rupture of membranes.

2. To establish the association between lactate levels in vaginal fluid and time of spontaneous onset of labour in cases of suspected prelabour rupture of membranes.

Study Design: In this prospective observational study 108 pregnant women with singleton pregnancy of more than 34 weeks of gestation, with prelabour rupture of membranes underwent a sterile speculum examination and vaginal fluid was taken to estimate lactate level. Lactate levels were analysed in Auto analyser using spinreact kit. Then, women were followed till spontaneous onset of labour.

Results: The median time of onset of spontaneous labour and examination was 12 hours in patients with lactate levels $>5.0 \mathrm{mmol} / \mathrm{L}$ and 72 hours in patients with lactate levels $<5.0 \mathrm{mmol} / \mathrm{L}$. Among 55 women with lactate levels $>5.0 \mathrm{mmol} / \mathrm{L}, 65.5 \%$ of women $(n=36)$ had spontaneous onset of labour within 24 hours and $85.5 \%$ of women $(n=47)$ within 48 hours. The odds ratio of being in labour within 24 hours is almost 18 times higher among women with lactate concentrations $>5.0 \mathrm{mmol} / \mathrm{L}$ than women with lower lactate levels.

Conclusion: A lactate level $>5.0 \mathrm{mmol} / \mathrm{L}$ is significantly associated with the spontaneous onset of labour within 24 - 48 hours in cases of suspected prelabour rupture of membranes.

Keywords: Lactate, Prelabour rupture of membranes, labour, NICU (neonatal intensive c

\section{Introduction}

Prelabour rupture of membranes is a syndrome characterized by rupture of the foetal membranes before labour ${ }^{1}$. It occurs in approximately $10 \%$ of all pregnancies and is implicated in more than one third of the preterm deliveries ${ }^{2}$. The time between the rupture of membranes and the onset of labour (latent period) may extend from hours to days ${ }^{1}$. PROM is associated with significant maternal risks including chorioamnionitis with serious systemic infection and neonatal morbidity and mortality including prematurity, sepsis and pulmonary hypoplasia. ${ }^{1}$ PROM accounts for $30 \%$ of instances of premature labour and $18-20 \%$ of prenatal mortality ${ }^{3}$. A very important question in clinical practice with regard to women with prelabour rupture of membranes is the time of spontaneous onset of labour. Latency, which is defined as the time of premature rupture of membranes to delivery, is highly variable. Latency is important in terms of referral of patients to higher institutions for appropriate management of high risks infants, administration of steroids and thus improve the perinatal outcome $^{2}$. Traditionally, the diagnosis of PROM has relied on combination of factors, including the woman's history, pooling of amniotic fluid in the vagina, microscopic examination of the vaginal fluid, biochemical tests.The biochemical tests includeNitrazine, Vaginal di-amine oxidase, Prolactin, Alpha fetoprotein, Insulinlike growth factor binding protein -1 , Human chorionic gonadotrophin, Fibronectin, AmniSure placental alpha microglobulin - 1.However, all these tests can diagnose prelabour rupture of membranes but none of these tests is able to determine the time of spontaneous onset of labour in women with complaints of suspected prelabour rupture of membranes. The absence of a "gold standard" has stimulated research for a clinically applicable marker to diagnose PROM as well as predict onset of labour so that adequate arrangements are made for the neonates.Lactate levels in vaginal fluid has been found to be such a marker. The lactate concentration in 
amniotic fluid is reported to be 4-6 times higher as compared with maternal and foetal blood ${ }^{2}$. The lactate concentration in vaginal fluid contaminated with amniotic fluid is found to be $0.8-15.6 \mathrm{mmol} / 1^{4}$. The source of lactate in amniotic fluid is the foetus (urine and lung excretion), the myometrium and choriodeciduaand its concentration in vaginal fluid is inversely proportional to time of spontaneous onset of labour. The measurement of lactate concentration in the vaginal fluid is an easy, inexpensive, rapid and non-invasive tool for diagnosis of prelabour rupture of membranes and also tells about latent period between prelabour rupture of membranes and time of spontaneous onset of labour. The present study was conducted to diagnose prelabour rupture of membranes and to establish the association between lactate levels in vaginal fluid and time of spontaneous onset of labour in cases of suspected prelabour rupture of membranes.

\section{Study Design And Methodology}

The present study was prospective observational study, conducted in the Postgraduate Department of Gynaecology and Obstetrics, LallaDed Hospital, Government Medical College Srinagar, in collaboration with the Department of Biochemistry, over a period of one and half year from April 2014 to September 2015 after obtaining ethical clearance from the institutional ethical committee. The study population included 108 pregnant women with singleton pregnancy of more than 34 weeks of gestation, with the suspicion of prelabour rupture of membranes. Written informed consent was taken and women fulfilling the inclusion criteria were divided into two groups on the basis of visibility of amniotic fluid:

A. Group 1 : Visible 43 patients

B. Group 2 : Non-visible 65 patients

Women with prelabour rupture of membranes who werealready in labour, having meconium stained liquor, with features of chorioamnionitis, women who were induced before the end of the latent phase or delivered by caesarean section before the end of latent phase and women with antepartum haemorrhage were excluded from the study. All the women in the study had undergone sterile speculum examination and 50 microlitres of vaginal fluid was collected in micro pipette for analysis of lactate levels. Lactate levels were analysed by kinetic method in Auto analyzer using spinreact kit. The levels were determined within 2-3 hours and if not possible, the sample was stored at $-20^{\circ} \mathrm{C}$ and the lactate levels were determined within 24- 48 hours. These patients were followed up for spontaneous onset of active phase of labour, which is defined as regular painful contractions of more than 3 in number lasting for 40 to 45 seconds within 10 minutes with cervical dilatation of 4 centimeters.

\section{Statistical Analysis}

Statistical Package for Social Sciences (SPSS - Version 20.0) and MedCalc software's were used to carry out the statistical analysis of data. Data was analysed by means of descriptive statistics viz., percentages, means and standard deviations. Graphically the data was presented by bar charts and pie charts. The association of lactate concentration and visibility of amniotic fluid with onset of labour was assessed by Chi-square test and Odds ratio. Parametric data was analysed with Student's independent t-test. P-value less than 0.05 was considered statistically significant.

\section{Observations And Results}

From April 2014 to September 2015, 108 women of gestational age of more than 34 weeks admitted with chief complaints of leaking per vaginumwere studied.The mean age of patients in visible group was $29.1 \pm 4.39$ years and in the non visible group was $29.4 \pm 4.68$ years. (Table 1) Most of the patients in both groups were Primigravidae $-67.4 \%$ in visible group and $67.6 \%$ in non visible group.(Table 2)The mean gestational age in visible group was $36.6 \pm 1.75$ weeks and in the non visible group was $36.1 \pm 1.80$ weeks.(Table 3 ) In this study only one patient in the visible group had lactate levels less than $5.0 \mathrm{mmol} / \mathrm{L}(2.3 \%)$ whereas $80 \%(52 / 65)$ of the non visible group had lactate levels less than 5.0mmol/l (Figure 1) Figure (2)shows36 out of 55 (65.5\%) patients with lactate levels of $\geq 5.0 \mathrm{mmol} / \mathrm{L}$ had spontaneous onset of labour within 24 hourswhere as only 5 of $53(9.4 \%)$ patients with lactate levels of $\leq 5.0 \mathrm{mmol} / \mathrm{L}$ had onset of labour within 24 hours. Figure (3) shows 47 out of 55 $(85.5 \%)$ women with lactate levels of $\geq 5.0 \mathrm{mmol} / \mathrm{L}$ had onset of labour within 48 hours where as only 11 out of $53(20.8 \%)$ patients with lactate levels $\leq 5.0 \mathrm{mmol} / \mathrm{L}$ had onset of labour within 48 hours. Figure (4) shows $58.1 \%$ (25 of 43) in the visible group had onset of labour within 24 hours where as only $24.6 \%$ (16 of 65) in the non-visible group had onset of labour within 24 hours. Figure (5) shows 83.7\% (36 of 43) in the visible group had onset of labour within 48hours where as 33.8\% (22 of 65) in the non visible group had onset of labour within 48 hours. With the lactate levels $\geq 5.0 \mathrm{mmol} / \mathrm{L}, 87.8 \%$ (36 of 41 ) of women had onset of labour within 24 hours while as $81.0 \%$ (47 of 58) of women had onset of labour within 48 hours. In the visible group, $60.9 \%$ (25 of 41) of women had onset of labour within 24 hours where as $62.1 \%$ (36 of 58) of women had onset of labour within 48 hours (Figure 6) 
Table 1: Distribution of patients as per age

\begin{tabular}{|l|l|l|l|l|l|}
\hline \multirow{2}{*}{ Age (years) } & \multicolumn{2}{|l|}{ Visible group $(\mathbf{n = 4 3 )}$} & \multicolumn{2}{l|}{ Nonvisible group $(\mathbf{n}=\mathbf{6 5})$} & \multirow{2}{*}{ P-value } \\
\cline { 2 - 5 } & No. & \%age & No. & \%age & 0.733 \\
\hline$\leq 24$ & 5 & $11.6 \%$ & 7 & $10.8 \%$ & $64.6 \%$ \\
\hline $25-34$ & 29 & $67.4 \%$ & 42 & $24.6 \%$ & \\
\hline$\geq 35$ & 9 & $20.9 \%$ & 16 & $29.4 \pm 4.68$ & \\
\hline Mean \pm SD & $29.1 \pm 4.39$ & & & \\
\hline
\end{tabular}

Table 2: Distribution of patients as per parity

\begin{tabular}{|l|l|l|l|l|}
\hline \multirow{2}{*}{ Parity } & Visible group $(\mathbf{n = 4 3})$ & \multicolumn{2}{l|}{ Nonvisible group $(\mathbf{n}=\mathbf{6 5})$} \\
\cline { 2 - 5 } & No. & \% ge & No. & age \\
\hline Primigravida & 29 & $67.4 \%$ & 42 & $65 \%$ \\
\hline Gravida $\geq 2$ & 14 & $32.6 \%$ & 23 & $35 \%$ \\
\hline P-value $=0.762$ (Not Sig.) & \multicolumn{4}{|l}{} \\
\hline
\end{tabular}

Table 3: Showing gestational age (weeks) of patients

\begin{tabular}{|l|l|l|l|l|l|}
\hline \multirow{2}{*}{ Gestational Age (weeks) } & \multicolumn{2}{|l|}{ Visible group $(\mathbf{n = 4 3})$} & \multicolumn{2}{|l|}{ Nonvisible group $(\mathbf{n = 6 5})$} & \multirow{2}{*}{ P-value } \\
\cline { 2 - 5 } & No. & \% ge & No. & \% age & \\
\hline $34-36$ & 13 & $30.2 \%$ & 31 & $48 \%$ & $25 \%$ \\
\hline $36-38$ & 14 & $32.6 \%$ & 16 & $28 \%$ & \\
\hline $38-40$ & 16 & $37.2 \%$ & 18 & $36.1 \pm 1.80$ & \\
\hline Mean \pm SD & $36.6 \pm 1.75$ & & & \\
\hline
\end{tabular}

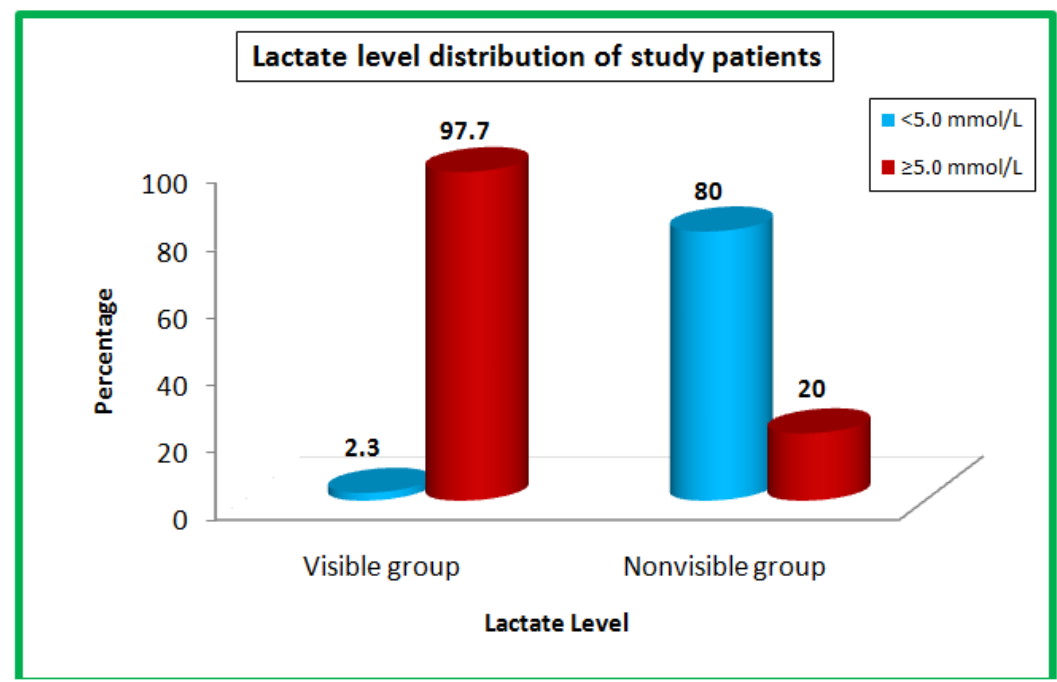

Figure 1

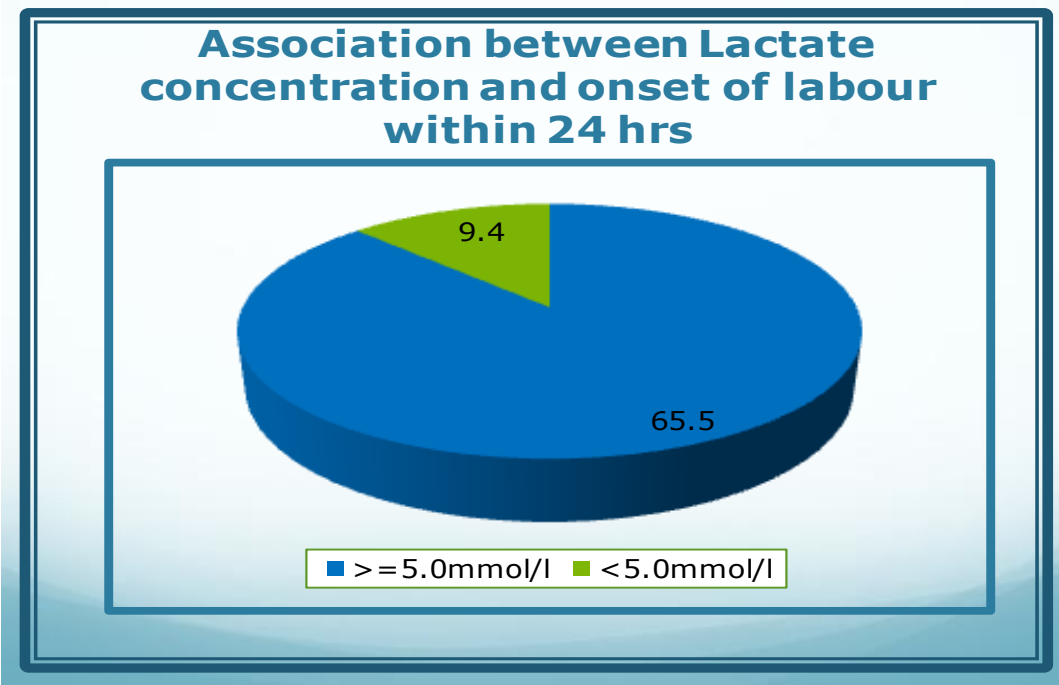

Figure 2 


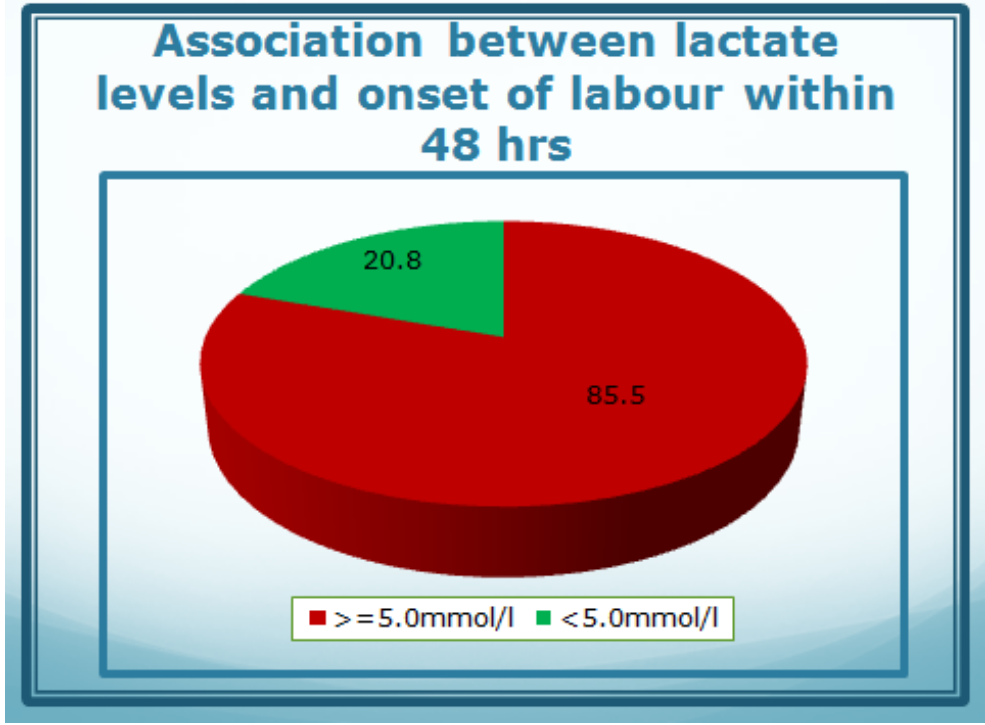

Figure 3

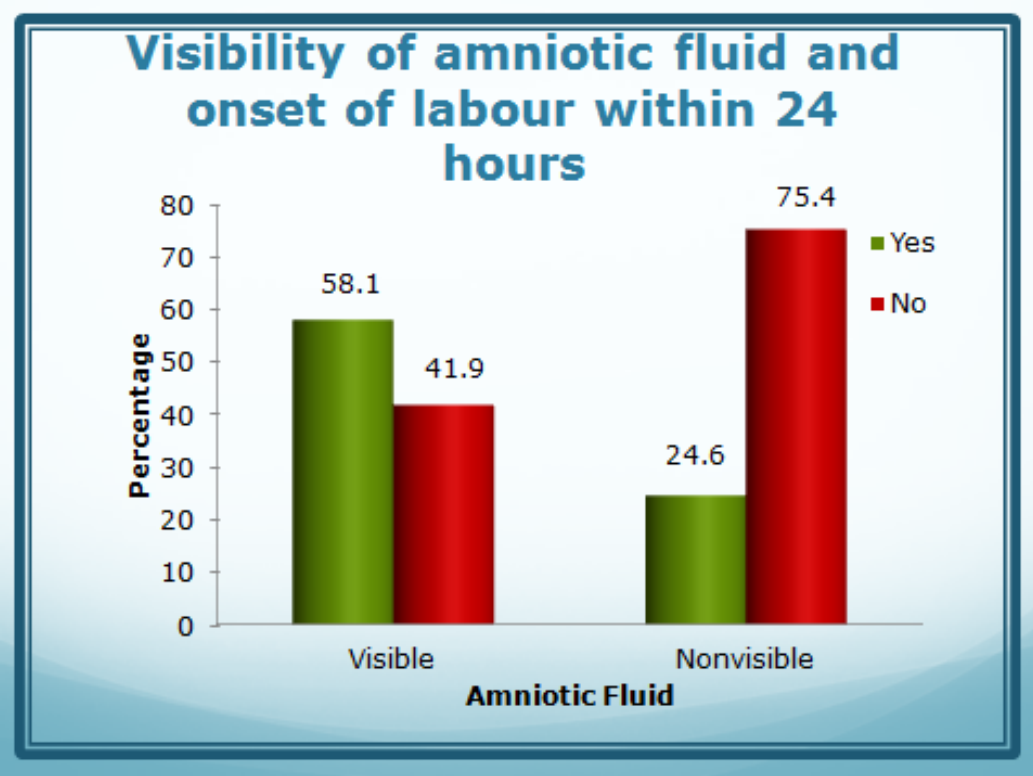

Figure 4

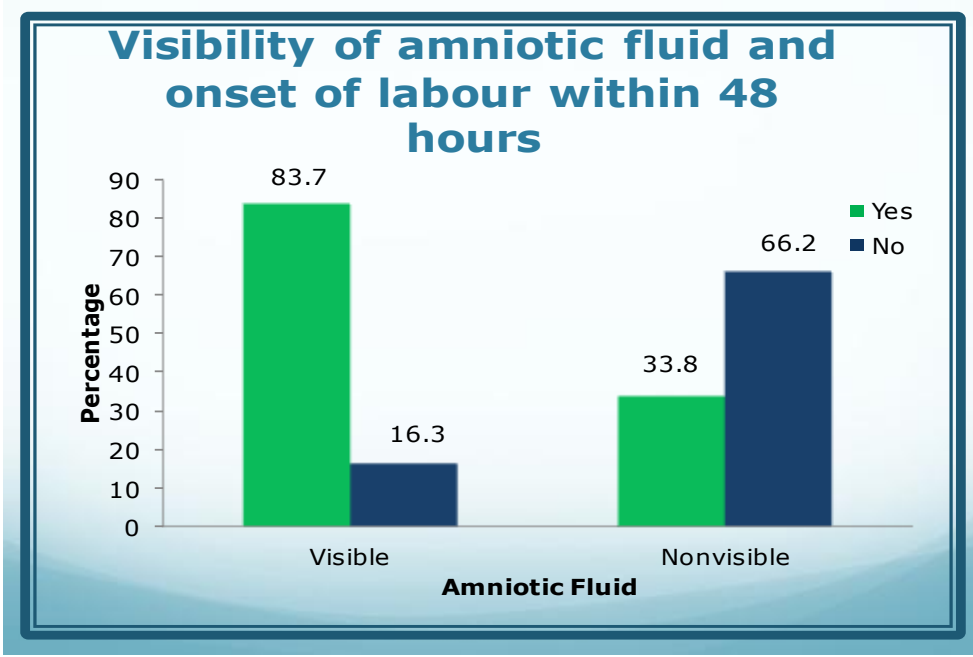

Figure 5 


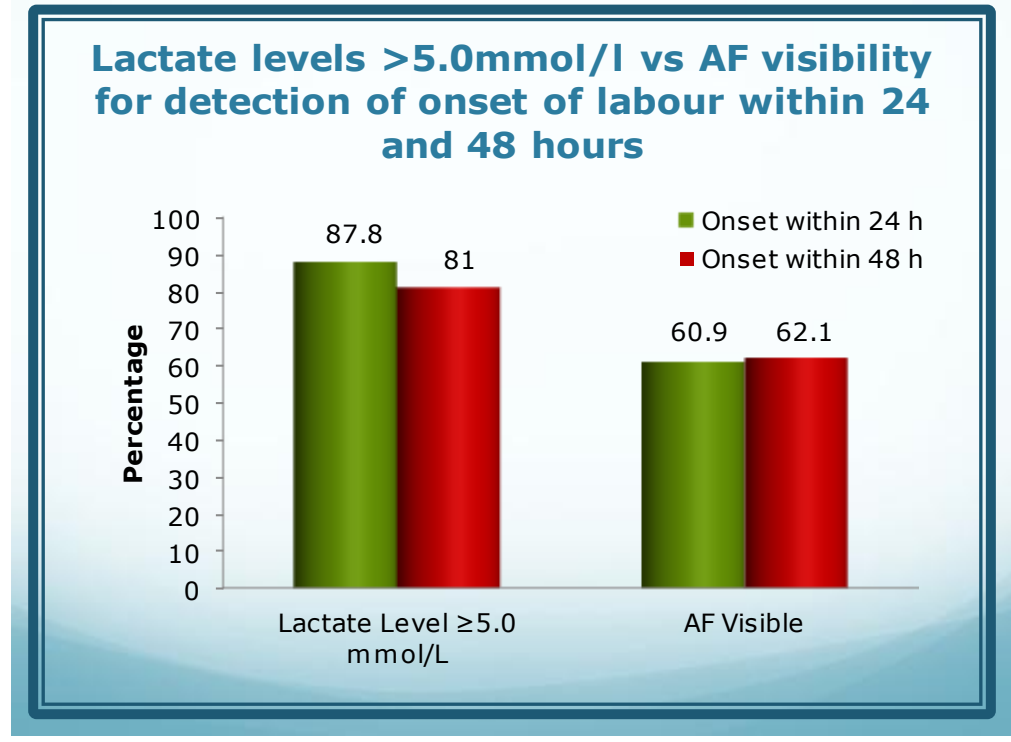

Figure 6

\section{Discussion}

The study was conducted in 108 pregnant women with gestational age $\geq 34$ weeks admitted in the hospital with chief complaints of leaking per vaginum from April 2014 to September 2015. In our study we found that the demographic profile of study population was almost similar in age, parity and gestational age in both visible and non visible groups which was comparable to the studies conducted byJaiswar S.P. et al (2013) ,NasrinNazari et al (2012), E.Wiberg-Itzel (2006) $)^{2,4,5}$. The mean age of patients in visible group was $29.1 \pm 4.39$ years and in thenon visible group was $29.4 \pm 4.68$ years. The mean gestational age in visible group was $36.6 \pm 1.75$ weeks and in the non visible group was $36.1 \pm 1.80$ weeks.Most of the patients in both groups were Primigravidae $-67.4 \%$ in visible group and $67.6 \%$ in non visible group. Regarding the distribution of various lactate levels in the study population, it was seen that only one patient in the visible group had lactate levels less than $5.0 \mathrm{mmol} / \mathrm{L}$ whereas $80 \%(52 / 65)$ of the non visible group had lactate levels less than $5.0 \mathrm{mmol} / 1$ which was comparable to the studies conducted byJaiswar S.P. et al (2013), El-Sokkary M. et al (2015)

In our study, the median time of onset of spontaneous labour in patients who had lactate levels of less than $5.0 \mathrm{mmol} / \mathrm{L}$ was 72 hours and in patients who had lactate levels of more than $5.0 \mathrm{mmol} / \mathrm{L}$ was 12 hours which was comparable to the studies conducted byJaiswar S.P. et al (2013), Wiberg- Itzel $\mathbf{t}$ al (2006) ${ }^{2,5}$.In our study, the odds ratio of being in labour within 24 hours was almost 18.2 times higher among women with lactate concentrations $>5.0 \mathrm{mmol} / \mathrm{L}$ than women with lower lactate levels. This was comparable to the study conducted byJaiswar S.P. et al (2013) ${ }^{2}$.

In our study, the odds ratio of being in labour within 48 hours was 22.4 times higher among women with lactate concentrations $>5.0 \mathrm{mmol} / \mathrm{L}$ than with lower lactate levels. Results were comparable to the studies conducted by Jaiswar S.P. et al (2013), Wiberg- Itzel t al (2006) $)^{2,5}$.

In our study, the odds ratio of being in labour within 24 hours was almost 4 times higher among women with visible amniotic fluid than in the non visible group which was comparable to the study conducted by Jaiswar S.P. et al (2013) ${ }^{2}$.

In our study, the odds ratio of being in labour within 48 hours was almost 10 times higher among women with visible amniotic fluid than in the non visible group. Results were comparable to the study conducted by Jaiswar S.P. et al (2013) ${ }^{2}$.

With the lactate levels of $\geq 5.0 \mathrm{mmol} / \mathrm{L}, 87.8 \%$ of patients had spontaneous onset of labour within 24 hours and $81.0 \%$ of patients had spontaneous onset of labour within 48 hours in our study. Results were almost similar to the study by Jaiswar S. P. et al (2013) ${ }^{2}$.

On the basis of visibility of the amniotic fluid , $60.9 \%$ of the patients had spontaneous onset of labour within 24 hours and $62.1 \%$ of the patients had spontaneous onset of labour within 48 hoursin our study.Results were almost similar to the study by Jaiswar S. P. et al (2013) ${ }^{2}$.

\section{Conclusion}

PROM is major cause of perinatal morbidity and mortality. It is associated with $20 \%$ to $30 \%$ of all preterm births and the prognosis is related primarily to gestational age and period of latency between rupture of membranes and onset of labour. A timely and accurate diagnosis of PROM and determination of latency period 
is critical to optimize the pregnancy outcome. We have found that the lactate concentration is a valid method for the prediction of onset of labour within 24 and 48 hours. A high lactate concentration is more strongly associated with spontaneous onset of labour than visible amniotic fluid. Positive lactate test (lactate levels $>5.0 \mathrm{mmol} / \mathrm{L}$ ) strongly predicts the time of onset of spontaneous labour within $24-48$ hours whereas negative lactate test (lactate levels $<5.0 \mathrm{mmol} / \mathrm{L}$ ) gives strong support that women are not having PROM and will not go in labour within 48 hours. The women could be sent back home and can wait for spontaneous onset of labour. The measurement of lactate concentration in the vaginal fluid is an easy, inexpensive, rapid and non -invasive tool for diagnosis of prelabour rupture of membranes and time of spontaneous onset of labour to optimize perinatal outcome and minimize neonatal morbidity.

\section{References}

[1] Fernando Arias, Shiresh N Daftary, Amarnath G Bhinde. Practical guide to high risk pregnancy and delivery.

[2] JaswarShyamPyari, Natu SM Gupta Amit, ChaurassiaShewta. Association between lactate levels in vaginal fluid and time of spontaneous onset of labour in suspected cases of prelabour rupture of membranes. The Journal of Obstetrics and Gynaecology of India 2013; 63(3): 182-185.

[3] NasrinNazari, MahbobehAhmadi, Mohammed Mazani, Hamid AlaviMajd, MansourehRefaei. The diagnostic value of lactate level in the vaginal fluid for determining the premature rupture of membranes. Journal of Society for Development in New Net Environment in B and H 2012; Vol. 6, No. 12.

[4] Eva Wiberg -Itzel, S. Cnattingius,L. Nordstrom. Lactate determination in vaginal fluid; a new method in the diagnosis of prelabour rupture of membranes. International Journal of Obstetrics and Gynaecology 2005 volume 112; 754-758.

[5] Wiberg - Itzel E, H. Petterson, S. Cnattingius, L. Nordstrom. Association between lactate in vaginal fluid and time of spontaneous onset of labour for women with suspected prelabour rupture of the membranes. BJOG2006;113:1426-30.

[6] El-Sokkary M., Anbar A., Wahba K., El-Mandouh M. Vaginal Fluid Lactate: A Method for the Diagnosis of Premature Rupture of Membrane. International Journal of Obstetrics and Gynaecology Research (IJOGR) 2015; Vol. 2, No.3: pp. 170-187. 